\title{
First direct imaging of giant pores of the Metal-Organic Framework MIL-101
}

\author{
O. I. Lebedev ${ }^{\mathrm{a}}$, F. Millange ${ }^{\mathrm{b} *}$, C. Serre ${ }^{\mathrm{b}}$, G. Van Tendeloo ${ }^{\mathrm{a}}$, G. Férey ${ }^{\mathrm{b}}$ \\ a. University of Antwerp, Groenenborgerlaan 171, B-2020 Antwerpen, Belgium. \\ b. Institut Lavoisier, UMR 8637, Université de Versailles St-Quentin-en-Yvelines, 45 Avenue des Etats-Unis F-78035. Versailles \\ Cedex, France. E-mail : millange@chimie.uvsq.fr \\ ‡ Institut Universitaire de France (Versailles) F-78035. Versailles Cedex, France.
}

Figure 1 Supplementary data. (a) Pentagonal window; (b) Hexagonal window; (c) and (d) The two types of mesoporous cages filled with free water molecules, which have been omitted for clarity.

Figure 2 Supplementary data. SEM images of the MIL-101. All MIL-101 crystals grow as nanocrystals with a nearly perfect octahedral shape. 


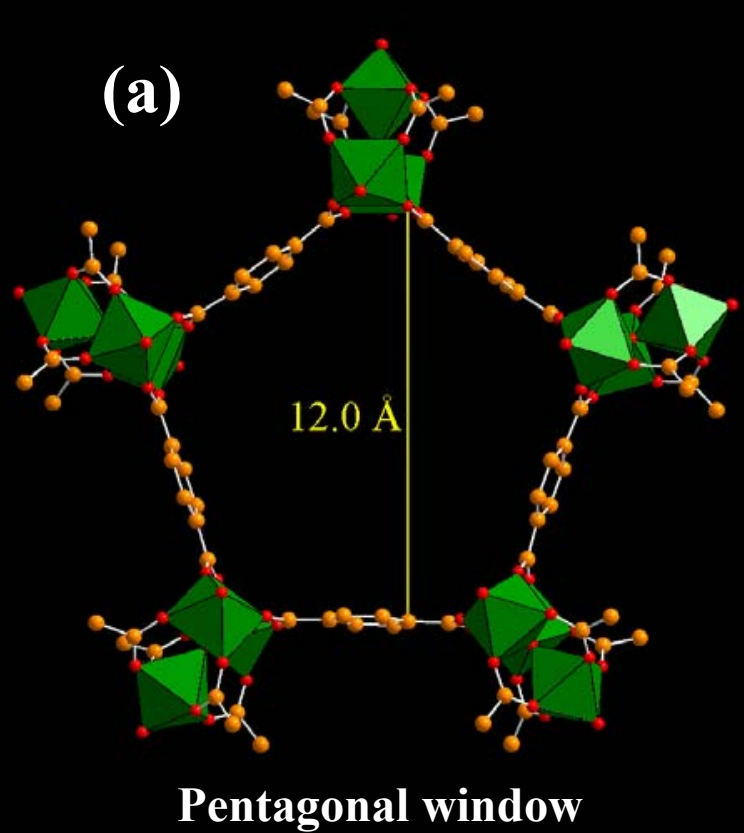

(b)

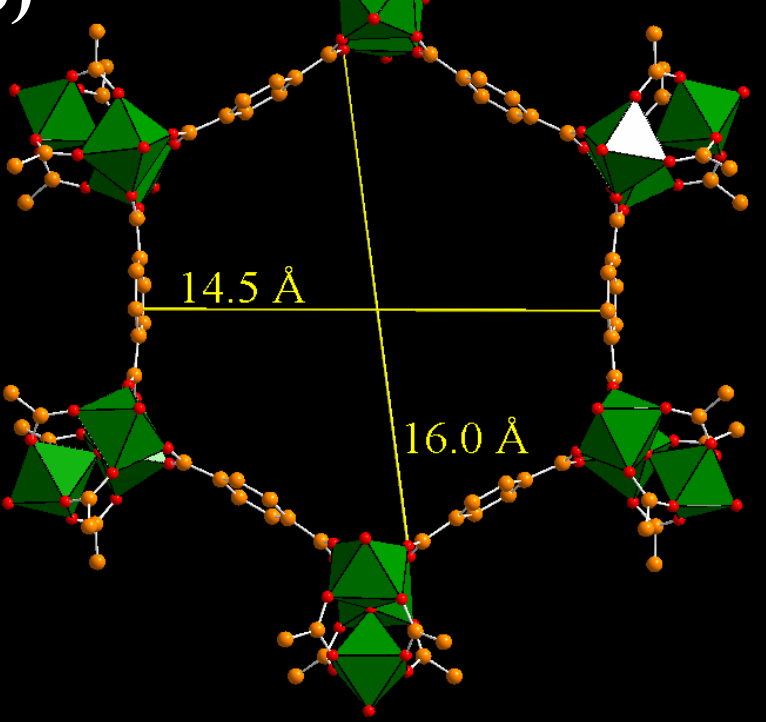

Hexagonal window

(c)

(d)

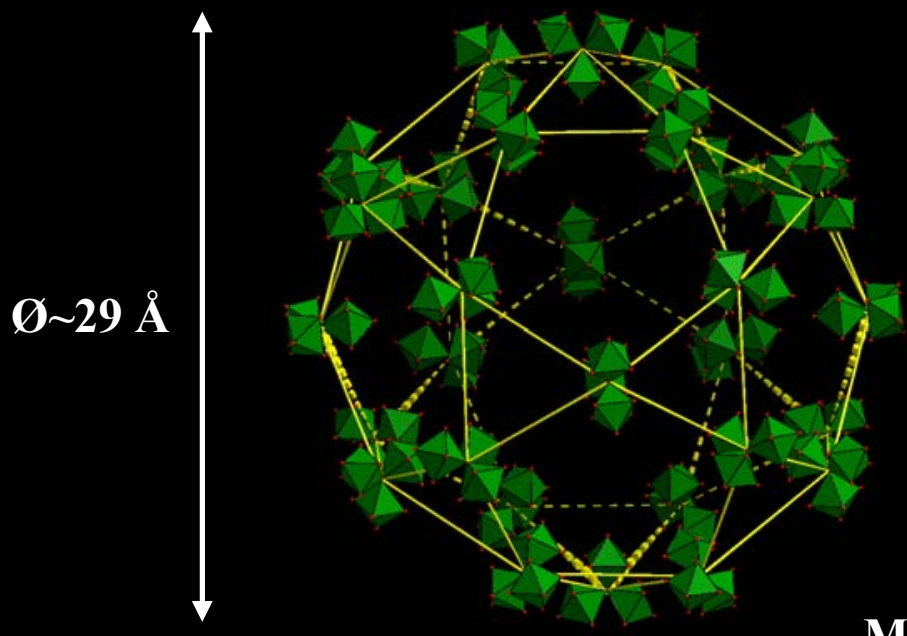

Mesoporous cages

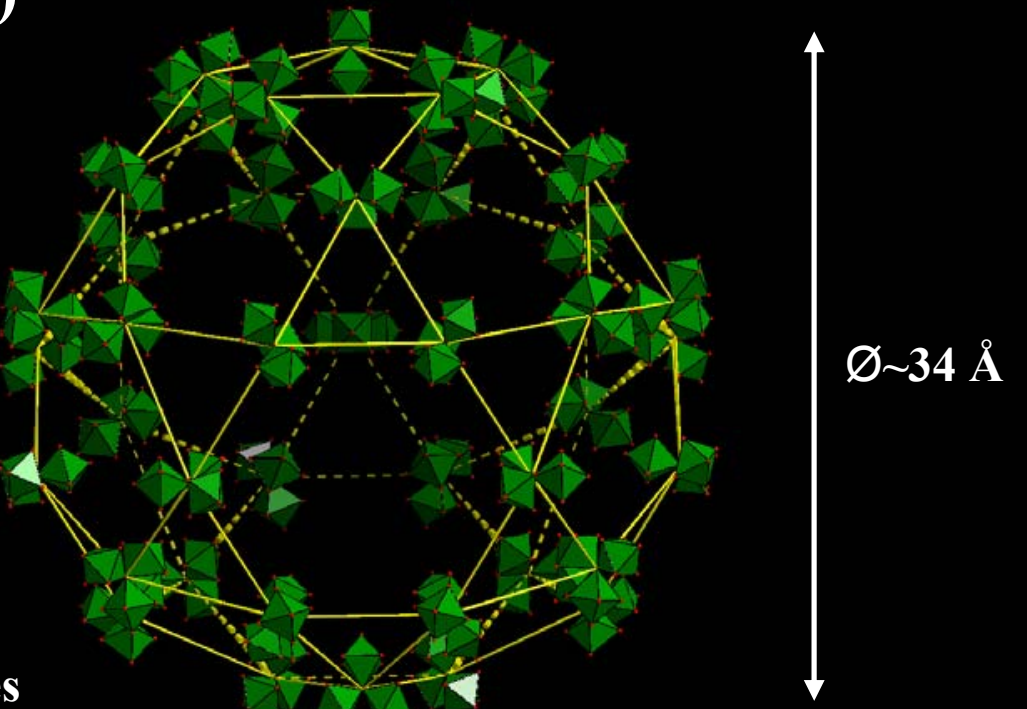

Figure 1 Supplementary data 


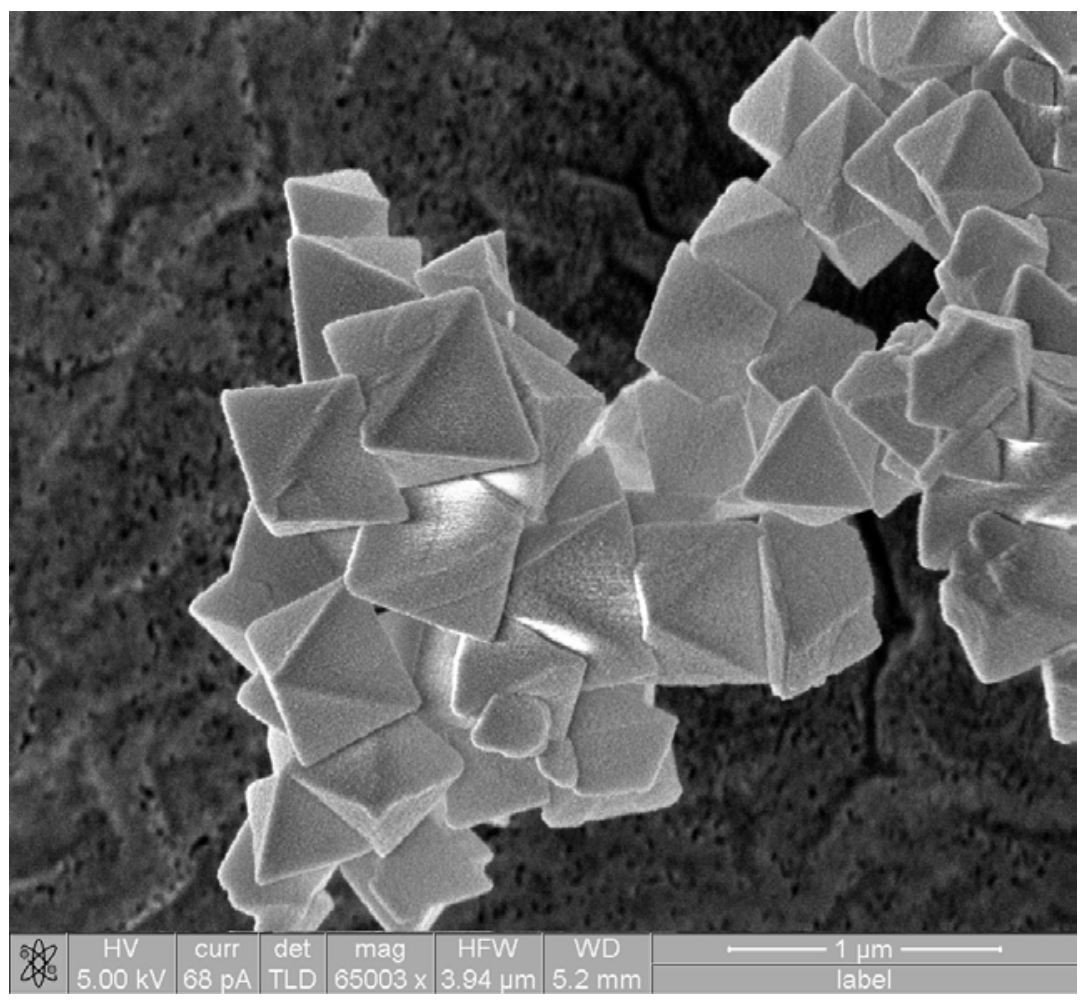

Figure 2 Supplementary data 